\title{
The impact of the multiple bile ducts on postoperative biliary complications in living donor liver transplantation: a single center experience
}

\author{
Doojin Kim, Doo-Hoo Lee, Sang-Tae Choi, Yeon Ho Park
}

Department of Surgery-Transplantation, Gachon University College of Medicine, Incheon, Korea

Background: The multiple bile ducts in a living donor graft are a long standing troublesome. All transplant surgeons try to avoid this problem due to technical difficulty and relation with biliary complications. However, there have been many mixed reviews about the impact of multiple bile ducts on biliary complications. This study aimed to investigate correlations between the number of bile duct and biliary complications in patients undergoing living donor liver transplantation (LDLT).

Methods: We reviewed all LDLT patients in our hospital between July 2008 and December 2020. The patients were divided into two groups according to the number of bile duct in living donor graft (single duct [SD], multiple duct [MD]). Biliary complications were defined by endoscopic, interventional, or surgical treatment. Collected data included demographics of donor and recipient, surgical data about to biliary anastomosis (duct-to-duct, hepaticojejunostomy, and conjoined), perioperative and postoperative outcomes.

Results: All 70 patients were represented SD $(n=48)$ and MD $(n=22)$. Biliary complications occurred in 27 patients $(38.6 \%)$ and were more common in MD group (54.5\% vs. 31.3\%). Although there were no statistic differences between two groups, multiple bile ducts was positively correlated with biliary complications (hazard ratio, 2.4). MD patients revealed longer operation time $(1,052 \pm 251$ vs. $910 \pm 215$ minutes; $P=0.019)$ and higher percentage of hepaticojejunostomy (31.8\% vs. $8.3 \%$; $P=0.012)$. Donor age, graft-recipient weight ratio, cold ischemic time and amount of transfusion were not different between two groups. Twenty-one (77.7\%) were fully recovered from biliary complications but three (4.3\%) resulted in graft failure.

Conclusions: Biliary complications were common problems in LDLT despite overall good result. Multiple bile ducts can be a potent risk factor of biliary complications among other predisposing factors.

Corresponding author: Doojin Kim

E-mail: drappendix70@gmail.com

This is an Open Access article distributed under the terms of the Creative Commons Attribution Non-Commercial License (http://creativecommons.org/licenses/by-nc/4.0/) which permits unrestricted non-commercial use, distribution, and reproduction in any medium, provided the original work is properly cited. 